\title{
Review: oral and intravaginal agents are equally effective for treatment of uncomplicated vulvovaginal candidiasis
}

\author{
Watson MC, Grimshaw JM, Bond CM, et al. Oral versus intra-vaginal imidazole and triazole anti-fungal treatment of \\ uncomplicated vulvovaginal candidiasis (thrush). Cochrane Database Syst Rev 2001;(4):CD002845 (latest version 28 May \\ 2001).
}
QUESTION: Are oral and intravaginal antifungal agents equally effective, safe, and cost effective for uncomplicated vulvovaginal candidiasis?

\begin{abstract}
Data sources
Randomised controlled trials (RCTs) published in any language were identified by searching the Cochrane Controlled Trials Register (CENTRAL/CCTR), the Cochrane Collaboration Sexually Transmitted Disease Group Specialised Register of Controlled Trials, EMBASE/Excerpta Medica (1980 to January 2000), and Medline (January 1985 to May 2000). Reference lists of each trial were reviewed, and UK manufacturers of antifungal agents were contacted.
\end{abstract}

\section{Study selection}

Trials were selected if they included women $\geqslant 16$ years of age with mycologically confirmed acute vulvovaginal candidiasis ( $<4$ episodes in $12 \mathrm{mo}$ ) and compared $\geqslant 1$ oral antifungal agent with an intravaginal antifungal agent. Trials were excluded if they included only participants who were HIV positive, immunocompromised, pregnant, breast feeding, or diabetic.

Source of funding: Chief Scientist Office, Scottish Executive Health Department, UK.

For correspondence: Dr M C Watson,

University of Aberdeen Aberdeen, Scotland, UK m.c.watson@abdn.ac.uk.

A modified version of this abstract also appears in

Evidence-Based Nursing.

\section{Data extraction}

Data were extracted on the type, dose, frequency, and duration of antifungal treatment; setting; participants; and outcome measures. Main outcomes were short term and long term clinical cure rates. Secondary outcomes included mycological cure rates (culture), incidence of adverse reactions, and cost effectiveness. Individual studies were assessed for methodological quality (random allocation, concealment of allocation, adequate follow up, and blinding of outcome assessors).

\section{Main results}

17 RCTs reporting 19 comparisons were included in the analysis. The trials assessed 2 oral agents (fluconazole and itraconazole) and 4 intravaginal agents (clotrimazole, econazole, miconazole, and terconazole).

Meta-analyses were done using a random effects model; the denominator for analysis was the number of randomised patients who had positive cultures for yeast before antifungal treatment began. Length of follow up was classified as short term ( 5 to $15 \mathrm{~d}$ ) and long term (2 to $12 \mathrm{wks})$. Oral and intravaginal antifungal agents did not differ for clinical cure at short term 9 comparisons, $\mathrm{n}=1247,80 \% \cup 80 \%)$ or long term (7 comparisons, $\mathrm{n}=836,83 \% v 82 \%$ ) follow up or for mycological cure at short term (17 comparisons, $\mathrm{n}=2239,83 \%$ v $82 \%$ ) or long term ( 14 comparisons, $\mathrm{n}=1711,72 \% v 66 \%$ ) follow up. Sensitivity analyses based on all randomised participants, blinding, and the proportion of patients followed did not change the effect sizes for any of the outcomes.

11 trials reported on adverse reactions. Intravaginal agents were associated with such local reactions as irritation, burning, and pruritis, and such systemic effects as headache, whereas oral agents were associated with such systemic effects as gastrointestinal effects and headache. Data were insufficient to compare the relative safety of oral and intravaginal agents. No trials of the relative cost effectiveness of oral and intravaginal agents were found.

\section{Conclusions}

Oral and intravaginal agents are equally effective in the treatment of uncomplicated vulvovaginal candidiasis. Insufficient data exist on adverse effects and cost effectiveness of the 2 types of treatment.

\section{COMMENTARY}

The findings of the systematic review by Watson $e t$ al support the general view of treatment for uncomplicated vulvovaginal candidiasis. The review did not identify any studies that compared single oral doses of antifungal agents with multidose oral antifungals, leaving this issue unexplored. Similarly, single topical applications were not compared with multidose topical applications. Efficacy - the cure rate under ideal clinical conditions - may differ from effectiveness - the cure rate in the real world (eg, forgotten applications and misapplications). The authors did not assess whether the efficacy and effectiveness of the antifungal agents measured in industry sponsored trials differed from those measured in non-industry sponsored trials.

Most vaginal discharges are caused by one of 3 organisms: a fungus (Candida), a protozoan (Trichomonas), and a combination of anaerobic and aerobic bacteria. Conditions caused by these organisms occur at different frequencies dependent on the population. In a middle-class US population, bacterial vaginosis occurs $50 \%$ of the time, and candidiasis and trichomonas each occur $25 \%$ of the time.

Concurrent partner treatment does not improve a woman's cure rate, ${ }^{2}{ }^{3}$ but, in the approximately $5 \%$ of women with recurrent infection, ${ }^{4}$ partner treatment to eradicate the intestinal reservoir of Candida has proved efficacious. ${ }^{5}$

The 17 RCTs were done in Europe (n=9), the USA ( $n=5)$, Japan $(n=1)$, Thailand $(n=1)$, and Africa $(n=1)$. The use of antifungal agents for vulvovaginal candidiasis varies by cultural habits, effectiveness, cost effectiveness, safety, and preferences characteristic of the given population.

Diane Harper, MD Dartmouth Medical School Lebanon, New Hampshire, USA

1 Mishell DR, Stenchever MA, Droegemueller W, et al, editors. Comprehensive gynecology. 3rd ed. St. Louis: Mosby, 1997.

2 Brundin J. The effect of miconazole on vulvo-vaginal candidosis in pregnant and non-pregnant women and their partners. Int J Gynaecol Obstet 1976;14:537-40.

3 Shihadeh AS, Nawafleh AN. The value of treating the male partner in vaginal candidiasis. Saudi Med J 2000;21:1065-7.

4 Ringdahl EN. Treatment of recurrent vulvovaginal candidiasis. Am Fam Physician 2000;61:3306-12, 3317.

5 Spinillo A, Carratta L, Pizzoli G, et al. Recurrent vaginal candidiasis. Results of a cohort study of sexual transmission and intestinal reservoir. $J$ Reprod Med 1992;37:343-7. 